community mental health clinicians as advocates for their pregnant clients, has been developed. Additional outcomes include an education package to up-skill clinicians in using the resource, a surveillance system to identify at-risk cases and an information system to enable evaluation of the impact on obstetric and child health outcomes.

Conclusion: This primary preventive intervention has the potential to significantly improve obstetric and neonatal outcomes for this high-risk cohort.

\section{Participatory action research: researching with disenfranchised populations}

\section{W Hill, T Koch}

University of Newcastle, Newcastle, Australia

Background: The aim of this study was to position participatory action research (PAR) as a vital, dynamic and relevant approach that can be used when researching disenfranchised populations. PAR enables people to contribute their ideas, and plan and partake in effective action to improve their lives.

Method: We have developed a chronic illness research program that has been guided by participatory principles. Research to date includes the following: the needs of people who were learning to live with human immunodeficiency virus (HIV) and the intrusion of fatigue, learning alongside older people with asthma, people (homeless men living with schizophrenia) who experienced incontinence, Aboriginal elders who wanted to develop strategies to bring their plight of the high incidence of diabetes to the attention of their community, and women who were homeless and had been sexually violated as children. In addition, PAR as a legitimate methodology, the literature identified the following disenfranchised groups: persons with chronic illnesses such as HIV/acquired immunodeficiency syndrome and depression $(n=26)$, psychiatric diagnoses (eg clinical depression, schizophrenia) $(n=15)$, survivors of abuse $(n=6)$, alcohol misuse $(n=2)$, illicit drug use $(n=4)$, prescription drugs use $(n=4)$, prison populations $(n=2)$ and people with organic brain disorders $(n=5)$.

Results/Conclusions: While we have used action research approaches to research with people to explore disruptive events and develop ways they can transition through the event to create a sense of continuity in their lives, the literature has established that PAR is now a well-established methodological means for engaging with disempowered and/or marginalized populations into their own health management.

\section{Validation of a pencil-and-paper measure for depression in the cardiac population: the DMI-10 and DMI-18}

\section{T Hilton, G Parker}

The Black Dog Institute, Sydney, Australia

Background: Two issues arise in relation to depression in the cardiac population. The first is in relation to clinical practice, although depression is now recognized as a risk factor for increased morbidity and mortality in those with acute coronary syndrome (ACS) and, to a lesser degree, heart failure (HF). It remains underdiagnosed and undertreated in clinical practice. The second issue is a measurement issue: much of the research on depression in the cardiac population relies on measurement of depression symptoms with psychiatric rating scales and applies cut-offs for these scales that were predetermined for use in psychiatric rather than medical setting. This may lead to measurement errors as many of these scales include somatic symptoms. A well-validated screening and measurement tool specifically validated for use in this population may speak to both these issues, aiding detection and treatment of this important risk factor, as well as measuring it more accurately for research purposes.

Methods: A total of 322 patients with ACS or HF completed the DMI measures, psychosocial questionnaires and a semistructured clinical interview during the hospital stay.

Results: Both measures showed good psychometric properties, with high sensitivity and specificity when evaluated against clinical judgment. Cut-off points of $\geq 6$ and $\geq 14$ were determined for the DMI- 10 and DMI-14, respectively.

Conclusion: The DMI-18 and DMI-10 are appropriate for use as screening instruments in cardiac patients.

\section{Neurological soft signs in schizophrenia: using transcranial magnetic stimulation to investigate motor overflow}

\section{K Hoy ${ }^{1,2}$, N Georgiou-Karistianis ${ }^{2}$, R Laycock ${ }^{3}$, P Fitzgerald ${ }^{1,2}$}

${ }^{1}$ Alfred Psychiatry Research Centre; ${ }^{2}$ Monash University, Melbourne, Australia; and ${ }^{3}$ La Trobe University, Bundoora, Victoria, Australia

Background: Neurological soft signs (NSSs) are impairments that indicate nonspecific cerebral dysfunction. NSSs are thought to be early premorbid traits of 